\title{
Controlling Josephson dynamics by strong microwave fields
}

\author{
B. Chesca, ${ }^{1,2}$ S. E. Savel'ev, ${ }^{1}$ A. L. Rakhmanov, ${ }^{1,3}$ H. J. H. Smilde, ${ }^{4}$ and H. Hilgenkamp ${ }^{4}$ \\ ${ }^{1}$ Department of Physics, Loughborough University, Loughborough LE11 3TU, United Kingdom \\ ${ }^{2}$ Physikalisches Institut-Experimentalphysik II, Universität Tübingen, Tubingen D-72076, Germany \\ ${ }^{3}$ Institute for Theoretical and Applied Electrodynamics, Russian Academy of Sciences, 125412 Moscow, Russia \\ ${ }^{4}$ Faculty of Science and Technology and Mesa +Institute of Nanotechnology, University of Twente, P.O. Box 217, \\ 7500AE Enschede, The Netherlands
}

(Received 25 June 2008; published 9 September 2008)

\begin{abstract}
We observe several sharp changes in the slope of the current-voltage characteristics (CVCs) of thin-film ramp-edge Josephson junctions between $\mathrm{YBa}_{2} \mathrm{Cu}_{3} \mathrm{O}_{7-\delta}$ and $\mathrm{Nb}$ when applying strong microwave fields. Such behavior indicates an intriguing Josephson dynamics associated with the switching from a parametric excitation regime induced by the magnetic field of the microwave via oscillations of the Josephson critical current to an ac-current-excitation regime triggered by the electric field of the microwave. We propose a model, which describes the observed features on the CVC in terms of microwave-induced multiple switching between running and locked solutions of sine-Gordon equation.
\end{abstract}

DOI: 10.1103/PhysRevB.78.094505

PACS number(s): 74.50.+r

\section{INTRODUCTION}

Josephson junctions are one of the main building blocks for superconducting electronics, including sensitive superconducting magnetometers, ${ }^{1}$ qubits, ${ }^{2}$ superconducting ratchets, amplifiers (e.g., see Refs. 3-5), superconducting terahertz emitters, ${ }^{6}$ and detectors (e.g., see Ref. 7). The recent growing interest to the studies of dynamics of Josephson Junctions was stimulated by proposals ${ }^{8-10}$ and realizations ${ }^{11-13}$ of several novel terahertz devices based on layered superconductors, which can be modeled as a stack of identical intrinsic Josephson junctions. A remarkable step toward the realization of superconducting terahertz electronics was the observation ${ }^{12}$ of terahertz radiation from mesa $\mathrm{Bi}_{2} \mathrm{Sr}_{2} \mathrm{CaCu}_{2} \mathrm{O}_{8+\delta}$ samples achieved for a certain operating points of current-voltage characteristics (CVCs). In strong contrast to earlier predictions (e.g., Ref. 8), this experiment ${ }^{12}$ shows that there is no need to apply any magnetic fields to achieve considerable terahertz radiation from Josephson junctions. However, the question of how magnetic fields affect terahertz emission remains widely open. Here we show that the magnetic field of the microwave (MW) strongly affects the CVC so that the resonant conditions for terahertz emission can be easily met.

For a single Josephson junction, as well as for a stack of identical junctions as in the experiments, ${ }^{12}$ with increasing dc bias current $I_{\mathrm{dc}}$, the CVC curve describes the so-called zero-voltage branch (where the voltage $V$ is almost zero) up to the critical current $I_{c}$ followed by a sudden jump to the resistive branch where $V \propto I_{\mathrm{dc}}$. When decreasing $I_{\mathrm{dc}}$, the voltage smoothly decreases and drops to zero when the dc bias current becomes smaller than a so-called return current $I_{r}$ $<I_{c}$ (Ref. 1) [see the CVC with no microwave in Fig. 1(a)]. For currents in the interval $I_{r}<I_{\mathrm{dc}}<I_{c}$, the CVC is characterized by a hysteresis; i.e., the $\mathrm{CVC}$ has two solutions. Interestingly, the strong radiation from the $\mathrm{Bi}_{2} \mathrm{Sr}_{2} \mathrm{CaCu}_{2} \mathrm{O}_{8+\delta}$ mesa was observed only at the resistive return branch of the CVC, which was associated to a special matching condition between the wavelength of the terahertz radiation and the sample size. ${ }^{12}$ Thus, to achieve a strong radiation from junctions we need to develop an approach allowing us to tune the CVC until resonance conditions are met. In this work we show experimentally that the control of the CVC can be achieved if a strong microwave signal is applied to a single junction. We observe that the hysteresis on the CVC is completely suppressed and that several shallow resonancelike structures are clearly visible on the CVC. As the microwave

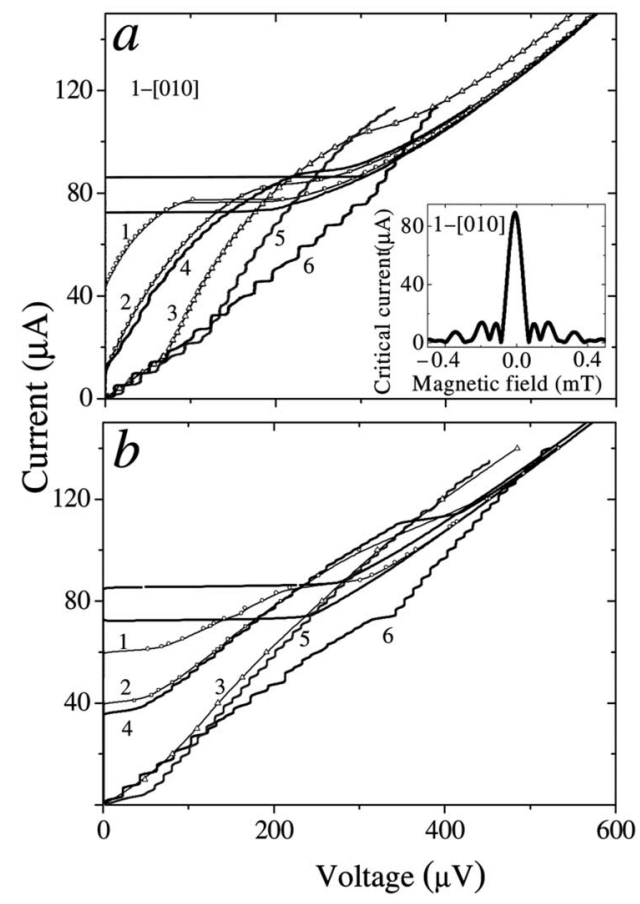

FIG. 1. The CVC for junction 1-[010] $\left[I_{c}(0)=86 \mu \mathrm{A}, f_{J}\right.$ $=104 \mathrm{GHz}$, and $R=3.85 \Omega]$ at different microwave powers. (a) Experiment and (b) calculations with $k=0.6$; no microwave for solid black lines with arrows; microwave with $f=2 \mathrm{GHz}$ (curves 1-3) and $f=5 \mathrm{GHz}$ (curves 4-6); microwave power $5 \mathrm{~dB}$ (curves 1 and 4), $10 \mathrm{~dB}$ (curves 2 and 5), and $15 \mathrm{~dB}$ (curve 3 and 6). Inset in (a) shows the measured dependence $I_{c}$ on $H_{\mathrm{dc}}$. 
power is increased the voltage position of such resonances increases monotonically. Such a behavior is in striking contrast to another microwave-induced effect that has been well investigated and understood, namely, the appearance of Shapiro steps ${ }^{1}$ on the CVC. Indeed, the Shapiro current resonances appear at voltages that are independent of the microwave power and are solely related to the microwave frequency. Then, the amplitude of Shapiro steps is only affected by the microwave power, in contrast to the case of the observed here shallow resonances. To understand Shapiro steps it is enough to assume that the microwave produces an alternating electric field of the same frequency across the junction which in turn induces an alternating current $I_{\mathrm{ac}}(t)$. To explain the formation of the observed unusual resonances, we assume instead that the microwave produces both an alternating electric field and an alternating magnetic field. The significance of the alternating magnetic field is that it induces oscillations of the Josephson critical current which appears to have crucial implications as far as the junction behavior in the presence of microwave is concerned. This model predicts the existence of three distinct dynamical regimes. (i) At low values of $I_{\mathrm{dc}}$, the system is mainly excited by the $H$-induced oscillations of the critical Josephson current $I_{c}[H(t)]$ occasionally switching the system to the running mode when $I_{c}$ temporally drops under the applied dc current. (ii) The other regime occurs when $I_{\mathrm{ac}}$, produced by the microwave electric field, is large enough to assist $I_{\mathrm{dc}}$ and drives the system from the locked state into the running state producing a fast increase in the voltage. (iii) The last regime occurs when $I_{\mathrm{dc}}$ is strong enough to keep the system in a running mode even when $I_{\mathrm{ac}}$ points in the opposite direction (i.e., $I_{\mathrm{dc}}-I_{\mathrm{ac}}>I_{r}$ ). At higher $I_{\mathrm{dc}}$, the system is always in the running mode asymptotically approaching the purely Ohmic regime. The model describes the observed features on the CVC and could be used to optimize the output of terahertz emitters.

\section{EXPERIMENT}

We prepared thin-film ramp-edge junctions between 170 $\mathrm{nm}$ untwinned $\mathrm{YBa}_{2} \mathrm{Cu}_{3} \mathrm{O}_{7-x}$ and $150 \mathrm{~nm} \mathrm{Nb}$ using a $30 \mathrm{~nm}$ $\mathrm{Au}$ barrier. The junctions are fabricated on the same chip, and the angle $\theta$ with the $\mathrm{YBa}_{2} \mathrm{Cu}_{3} \mathrm{O}_{7-x}$ crystal $b$ axis is varied in units of $5^{\circ}$ (see Fig. 1 in Ref. 14). This allows us to study the effect of microwave radiation on the CVCs for $360^{\circ} / 5^{\circ}=72$ different tunneling directions in the $a b$ plane. It should be noted that due to the $d$-wave anisotropy of the superconducting order parameter in the $\mathrm{YBa}_{2} \mathrm{Cu}_{3} \mathrm{O}_{7-x}$ both quasiparticle resistivity $R$ and maximum superconducting Josephson current $I_{c}$ are strongly dependent on the tunneling direction in the $a b$ plane. ${ }^{14}$ It follows that this particular type of junction offers a unique opportunity to actually look into how microwave radiation affects CVC for various junction parameters (i.e., $R$ and $I_{c}$ ) within a wide range. This explains our choice for the $\mathrm{Nb} / \mathrm{YBa}_{2} \mathrm{Cu}_{3} \mathrm{O}_{7-x}$ hybrid junctions in these experiments. Microwave influence on CVCs versus tunneling direction will be discussed elsewhere.

Our experimental setup allows us to significantly change McCumber parameter that mimics the evolution of the Josephson current $J_{c}$ a stack of intrinsic junction near the so- called vortex melting line, where $J_{c}$ strongly varies. The situation studied here (the case of a single junction) could be seen as an ideal behavior of Josephson junctions in strong microwave field when it is not smeared by complex collective dynamics of coupled intrinsic junctions. Indeed, the Josephson dynamics of intrinsic junctions are more complicated: the synchronization-desynchronization transition of intrinsic Josephson junctions strongly affects their CVC as well. ${ }^{15}$ A separate investigation would be of interest to perform in order to understand how both the electrical and magnetic fields of strong microwave radiation would affect CVCs of stacked intrinsic Josephson junctions.

In this work we are considering $\mathrm{YBa}_{2} \mathrm{Cu}_{3} \mathrm{O}_{7-x} / \mathrm{Nb}$ junctions for which tunneling occur in the $a b$ plane either in the [010] or in the [100] direction. A total of eight junctions have been measured, all being $4 \mu \mathrm{m}$ wide. So far, there have been several reports on these junctions that address junction fabrication, ${ }^{16}$ superconducting order parameter issues, ${ }^{16} \mathrm{An}$ dreev bound states-induced zero-bias anomaly in the quasiparticle tunneling, ${ }^{17}$ and microwave-induced Shapiro steps. ${ }^{18}$ To check the Josephson current homogeneity along the junction width we first investigated the dependence of the Josephson critical current, $I_{c}\left(H_{\mathrm{dc}}\right)$, on a dc magnetic field $H_{\mathrm{dc}}$ applied along the [001] direction. The junctions have an $I_{c}\left(H_{\mathrm{dc}}\right)$ that qualitatively resembles a Fraunhofer pattern [see the inset in Fig. 1(a)], suggesting a homogenous distribution of the critical current density $j_{c}$ along the junctions.

Next, we will consider the properties of the junctions under the influence of an applied microwave radiation. The microwave is applied via a high-quality coaxial cable whose open end is in close proximity (a few of millimeters) to our junctions. We are going to focus on two representative junctions only for which tunneling occurs along the [010] direction (further referred as 1-[010] and 2-[010]). Two qualitatively different behaviors are observed. First behavior is for microwave frequency $f$ in the range $0.01<f / f_{J}<0.1$ in which case Shapiro steps appear at integer $n$ multiples of the voltage $V_{0}$, satisfying the Josephson voltage-frequency relation $f / V_{0}=0.486 \mathrm{GHz} / \mu \mathrm{V}$. Here $f_{J}=\omega_{J} / 2 \pi=\sqrt{e I_{c} / 2 \pi^{2} \hbar C}$ is the characteristic (Josephson) plasma frequency of the junction (typically of about $100 \mathrm{GHz}$ ) and $C$ is the capacity of the contact. This behavior is well understood ${ }^{1}$ and it will not be the subject of further investigation in this work. It has to be added, however, that no trace of half-integer Shapiro steps at multiples of $V_{0} / 2$ were observed strongly suggesting a purely sinusoidal current-phase relation for the Josephson junctions. ${ }^{18}$ The latter observation along with the conclusion about homogeneity of the Josephson current along the junction width allows us to apply the usual sine-Gordon equation for the analysis of CVC in our samples. A second microwave-induced behavior can be summarized as follows. With increasing microwave power, the CVC of the junction changes drastically [see Figs. 1(a), 2(a), and 3(b)]: the hysteresis is strongly suppressed, and some intriguing structures that includes sharp changes in the CVC slope appear (which are reminiscent of shallow resonances). In contrast to the case of Shapiro steps (see, e.g., Fig. 1) which are located at equidistant voltages that are independent of the amplitude of the microwave power, the voltage position of the obtained structures on the CVC monotonically increases with the mi- 


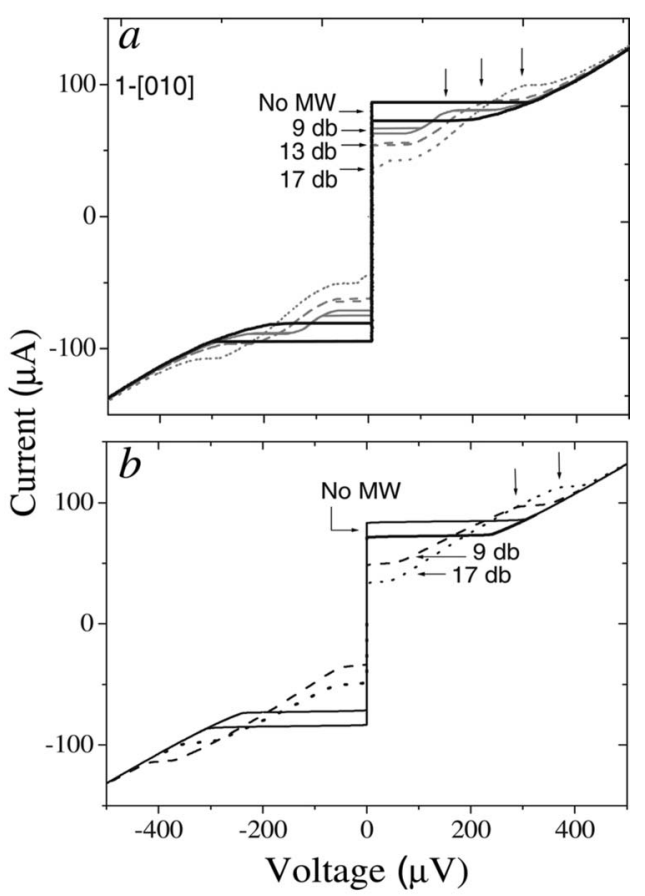

FIG. 2. The CVC for junction 1-[010] at different MW powers; the vertical arrows show the peak voltage position of the resonancelike structures. (a) Experimental curves at $f=0.5 \mathrm{GHz}$ and (b) calculated curves for $k=0.6$.

crowave power. Thus, as microwave power increases the peak position of the first resonant-like structure shifts in the direction indicated by the big arrows in Fig. 3. Then, as shown by the vertical arrows in Figs. 2 and 3 the peak voltage position of the second resonancelike structure shifts toward higher values as well. These resonancelike structures are very robust against a change in $f$ in the range 0.001 $<f / f_{J}<0.1$ and are most pronounced at lower frequencies in the range $0.001<f / f_{J}<0.01$ where Shapiro steps are not observed anymore. To our knowledge, such structures on CVC have never been investigated so far.

\section{THEORY}

We consider a single Josephson junction at $-D<x<D$ in the $x$ direction. In the $y$ direction the contact is smaller, $L_{y}$ $\ll D$. The equation for the gauge-invariant phase difference $\varphi$ in the junction in the dimensionless variables can be written as $^{1}$

$$
\ddot{\varphi}+\dot{\varphi} / \sqrt{\beta_{c}}+\sin \varphi=\varphi^{\prime \prime}
$$

where the dots above the variables mean the differentiation over dimensionless time $\tau=\omega_{J} t$, the prime is the differentiation over dimensionless coordinate $\eta=x / \lambda_{J}, \beta_{c}=\hbar / 2 e I_{c} R^{2} C$ is the McCumber parameter, ${ }^{1} \lambda_{J}=\sqrt{\hbar c^{2} / 8 \pi e \Lambda j_{c}}$ is the Josephson length, $\Lambda=\lambda_{1}+\lambda_{2}, \lambda_{1,2}$ are the London depths in the left and right superconductors, and $R$ is the junction resistance. We assume that the contact is small and $2 D \leqq \lambda_{J}$; then, $I_{c}=4 D L_{y} j_{c}$. The boundary conditions to Eq. (1) are

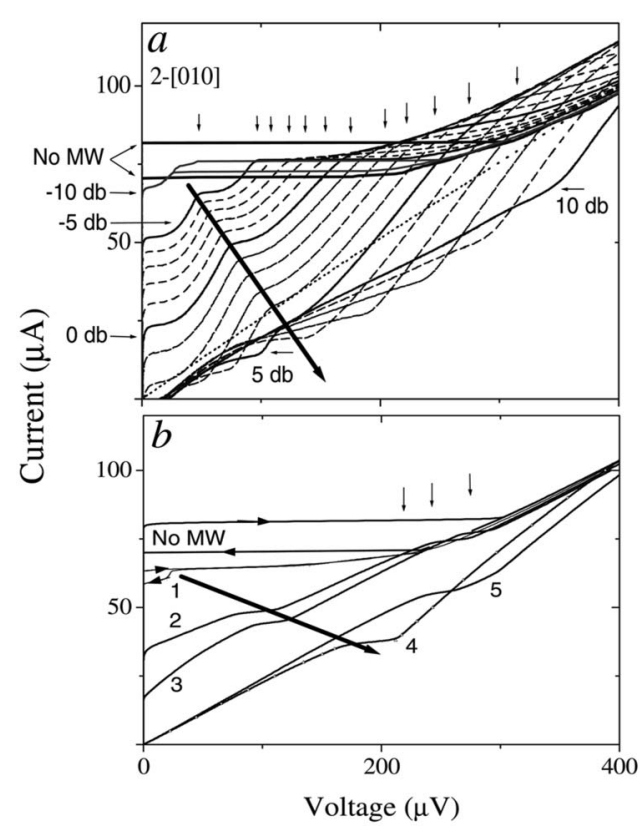

FIG. 3. The CVC for junction 2-[010] $\left[I_{c}(0)=82 \mu \mathrm{A}, f_{J}\right.$ $=108 \mathrm{GHz}$, and $R=4 \Omega]$ at different applied microwave powers. With increasing microwave power the peak position of the first resonantlike structure shifts in the direction indicated by the big arrows. The vertical arrows show the peak voltage position of the second resonancelike structure. (a) Experimental curves. Microwave power increases by $1 \mathrm{~dB}$ from -5 to $10 \mathrm{~dB}$. (b) Calculated CVC: (1) $-10 \mathrm{~dB}$ and $k=0.02$, (2) $0 \mathrm{~dB}$ and $k=0.02$, (3) $4 \mathrm{~dB}$ and $k=0.02$, (4) $7 \mathrm{~dB}$ and $k=0.1$, (5) $10 \mathrm{~dB}$ and $k=0.1$, and dashed line $-20 \mathrm{~dB}$ and $k=0.1$.

$$
\varphi^{\prime}=\frac{c H}{4 \pi j_{c} \lambda_{J}} \pm \frac{I}{4 j_{c} \lambda_{J} L_{y}} \text { at } \eta= \pm d,
$$

where $H$ is the $y$ component of the applied microwave magnetic field, $I$ is the total current through the contact, and $d$ $=D / \lambda_{J}$. Introducing the dimensionless field

$$
h(\tau)=\frac{c H(\tau)}{4 \pi j_{c} \lambda_{J}}
$$

and the current

$$
i(\tau)=I(\tau) / I_{c},
$$

we rewrite the boundary conditions as

$$
\partial \varphi / \partial \eta=h \pm i d, \quad \text { at } \quad \eta= \pm d .
$$

We seek $\varphi$ as a sum $\varphi=\psi+\chi$ of even, $\psi$, and odd, $\chi$, terms. Separating even and odd functions in Eq. (1), we derive

$$
\begin{aligned}
& \ddot{\psi}+\dot{\psi} / \sqrt{\beta_{c}}+\cos \chi \sin \psi=\psi^{\prime \prime}, \\
& \ddot{\chi}+\dot{\chi} / \sqrt{\beta_{c}}+\cos \psi \sin \chi=\chi^{\prime \prime},
\end{aligned}
$$

with the boundary conditions

$$
\frac{\partial \psi}{\partial \eta}= \pm i d
$$

and 


$$
\frac{\partial \chi}{\partial \eta}=h
$$

at $\eta= \pm d$. At low frequencies, $f \ll f_{J}$, and $d \lesssim 1$ (which corresponds the experimental conditions), the even solution $\psi(\eta)$ can be approximated as a sum of the coordinateindependent term $\psi$ plus $j \eta^{2} / 2<1$. Then, integrating Eq. (6) over $\eta$, we derive

$$
\ddot{\psi}+\dot{\psi} / \sqrt{\beta_{c}}+J(\tau) \sin \psi=i(\tau),
$$

where the value

$$
J(\tau)=\frac{1}{2 d} \int_{-d}^{d} d \eta \cos \chi(\eta, \tau)
$$

can be interpreted as a dimensionless critical current modulated by the microwave magnetic field. Under the condition $f \ll f_{J}$, we neglect the time derivative in Eq. (7) and obtain the first integral of this equation in the form

$$
\chi^{\prime 2}=h^{2}-2 \cos \psi \cos \chi .
$$

In the case $h^{2} \gg 1$, we have

$$
\chi=h(\tau) \eta
$$

and

$$
J(\tau)=\frac{\sin [h(\tau) d]}{h(\tau) d} .
$$

We assume that the microwave electric field

$$
E(t)=E \cos (2 \pi f t)
$$

induces an ac current in the sample

$$
I_{\mathrm{ac}}(t)=I_{\mathrm{ac}} \cos (2 \pi f t)
$$

and the amplitude of this current $I_{\mathrm{ac}} \propto E \propto H$, i.e.,

$$
I_{\mathrm{ac}}=k H \text {. }
$$

The coupling constant $k$ is determined by the circuit parameters and depends, in general, on $f, I_{\mathrm{dc}}$, and $H$. So, the current flowing through the junction is the sum

$$
I=I_{\mathrm{dc}}+I_{\mathrm{ac}}(t) .
$$

The instantaneous voltage, $V$, in the junction can be found from the standard Josephson relation, ${ }^{1}$

$$
2 e V(t)=\hbar \frac{d \varphi}{d t}
$$

In terms of $\varphi$ the averaged voltage (which was measured) reads

$$
V=\Phi_{0} \omega_{J}\left\langle\frac{d \psi}{d \tau}\right\rangle,
$$

where $\langle\cdots\rangle$ means time average. We compute $V=V\left(I_{\mathrm{dc}}\right)$ of the junction by solving Eq. (10) numerically. The results of the simulations are shown in Figs. 1(b), 2(b), and 3(c). In the calculations the values of all the sample parameters are determined from the measurements. However, the coupling

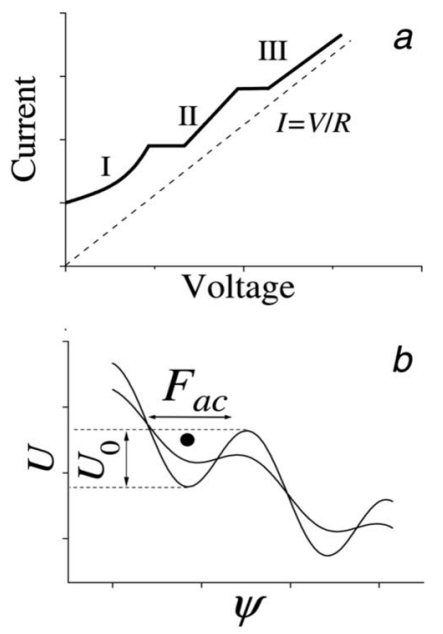

FIG. 4. (a) Schematic CVC of the junction under microwave irradiation. (b) Analogy with a driven junction: a particle in a cosine potential $U(\psi)$.

constant $k$ and numerical value of the microwave field amplitude $H$ could not be found accurately so that they are used as fitting parameters. The relative values of $H$ for different CVCs are kept the same as in the experiments.

\section{THREE DYNAMICAL REGIMES}

The computations reveal that the CVC curve exhibits three distinct regimes schematically shown in Fig. 4(a). First, at low dc bias current, the dynamics of the junction is determined by the parametric excitation due to oscillations of $I_{c}(H(t))$ (i.e., the modulation of the effective critical current). The dc average voltage across the junction occurs only when the effective Josephson critical current $I_{c}(t)$ (or $J(\tau)$ [in dimensionless units], see Eq. (11)) drops below $I_{\mathrm{dc}}+I_{\mathrm{ac}}$. This provides the initial increase in the voltage at low dc bias currents [the CVC part I schematically shown in Fig. 4(a)]. Using the well-known analogy between the Josephson dynamics and a particle moving on a cosine potential along the trajectory $\psi(t)$, this regime can be seen as modulations of the potential barriers $U_{0}(t) \cos (\psi)$ [see Fig. 4(b)]. The particle subject to a dc force starts moving when $U_{0}(t)<F_{\mathrm{dc}}$. Regime II sketched in Fig. 4(a) arises when the maximum possible current, $I_{\mathrm{dc}}+I_{\mathrm{ac}}$, reaches the value of the critical current $I_{c}$ producing a voltage jump in the CVC (however, the minimum current $I_{\mathrm{dc}}-I_{\mathrm{ac}}$ is smaller than the return current $I_{r}$ ). Within the model of a particle moving along a cosine potential, this occurs when the ac force $F_{\text {ac }}$ superposed on the dc force $F_{\mathrm{dc}}$ can push the particle over the highest possible potential barrier $U_{0}$. This produces a fast drift of the particle and, thus, a fast increase in the average voltage. However, the running particle can be eventually blocked when the ac force, pointed oppositely to the dc force, slows down the particle motion (which corresponds the condition $I_{\mathrm{dc}}-I_{\mathrm{ac}}$ $<I_{r}$ ). Consequently, the particle will be at rest for a while until the direction of ac force will be flipped to push it over the barrier. Regime III shown in Fig. 4(a) starts with a voltage jump when the difference $I_{\mathrm{dc}}-I_{\mathrm{ac}}$ exceeds the return cur- 
rent $I_{r}$. Again within the model of a particle moving along a cosine potential, this means that the dc force is so strong that the ac force cannot overcome $F_{\mathrm{dc}}$ and the particle is stopped. With a further increase in the dc bias current (dc force), the system approaches asymptotically the purely resistive Ohmic regime (where the effect of the potential on the particle motion is negligible). The switching between the first, second, and third dynamical regimes allows a significant modification of the CVC curve. Note that all these regimes are observed in the experiment [Figs. 1(a), 2(a), and 3(a)].

\section{CONTROLLING CURRENT-VOLTAGE CHARACTERISTICS}

The influence of the relatively high-frequency microwave radiation on the CVC is presented in Fig. 1. The microwave radiation suppresses the hysteresis. The Shapiro steps not seen at $2 \mathrm{GHz}$ are clearly visible at a higher $(5 \mathrm{GHz})$ frequency. The amplitude of the Shapiro steps changes with increasing of the microwave power while their positions are fixed. The curves for both frequencies shift right with the increase in the microwave power and start to cross (this behavior is the first manifestation of the peculiar Josephson dynamics mentioned above). Our simulations reproduce the characteristic features of the experimental curves including the very delicate crossings of CVC curves at different amplitudes and the disappearance of the Shapiro steps with the decrease in the frequency and/or power of microwave. The CVC at a lower frequency $(0.5 \mathrm{GHz})$ are shown in Figs. 2 and 3 for two different junctions. The influence of the Josephson dynamics on the CVC is even more pronounced here. Comparing the experimental and theoretical curves in Fig. 2, we can see a reasonable agreement: the hysteresis is suppressed by the microwave radiation, the curves intersect each other, and the resonancelike structures are shifted toward higher voltages with increasing microwave power.

\section{CONCLUSIONS}

In conclusion, we experimentally observed some microwave-induced resonant-like structures on the dc CVC of thin-film ramp-edge Josephson junctions made between $\mathrm{YBa}_{2} \mathrm{Cu}_{3} \mathrm{O}_{7-\delta}$ and $\mathrm{Nb}$. The unusual resonances have their voltage position on the CVC dependence on the microwave power rather than its frequency, while their amplitude is largely unaffected by an increase in the microwave power. As the dc bias current is increased one can distinguish three distinct regimes. All of these regimes can be qualitatively described within the sine-Gordon model taking into account both an electric field of microwave producing an ac bias current and a microwave magnetic field suppressing the Josephson critical current. The obtained results suggest a way to control the shape of the $\mathrm{CVC}$, allowing us to reach voltages within a considerable wide range at a given dc bias current. This control can be potentially useful for the recently proposed and realized terahertz emitters to fit necessary resonance conditions. Moreover, as suggested by our theoretical analysis the obtained effects are not restricted to a particular type of junction $\left(\mathrm{YBa}_{2} \mathrm{Cu}_{3} \mathrm{O}_{7-x} / \mathrm{Nb}\right)$ and should be observed for any type of Josephson junctions (including, e.g., a junction of two low $T_{c}$ superconductors or intrinsic Josephson junctions) under the influence of a strong microwave radiation. Note that in order to observe the discussed effect, the contact should be slightly underdamped and the applied microwave field should be strong enough to significantly affect the Josephson critical current. This was exactly the case for our experimental conditions.

\section{ACKNOWLEDGMENTS}

We acknowledge partial support from JSPS-RFBR Grant No. 06-02-91200, RFBR Grant No. 06-02-16691, ESF AQDJJ programs, and the EPSRC-GB via Grants No. EP/ D072581/1 and No. EP/F005482/1.
${ }^{1}$ A. Barone and G. Paterno, Physics and Applications of the Josephson Effect (Wiley, New York, 1982).

${ }^{2}$ J. Q. You and F. Nori, Phys. Today 58 (11), 42 (2005).

${ }^{3}$ M. Beck, E. Goldobin, M. Neuhaus, M. Siegel, R. Kleiner, and D. Koelle, Phys. Rev. Lett. 95, 090603 (2005).

${ }^{4}$ F. Marchesoni, S. Savel'ev, and F. Nori, Phys. Rev. E 73, 021102 (2006); S. Savel'ev, A. L. Rakhmanov, and F. Nori, ibid. 72, 056136 (2005).

${ }^{5}$ I. Zapata, R. Bartussek, F. Sols, and P. Hänggi, Phys. Rev. Lett. 77, 2292 (1996).

${ }^{6}$ G. Hechtfischer, R. Kleiner, A. V. Ustinov, and P. Müller, Phys. Rev. Lett. 79, 1365 (1997).

${ }^{7}$ V. P. Koshelets and S. V. Shitov, Supercond. Sci. Technol. 13, R53 (2000).

${ }^{8}$ M. Tachiki, M. Iizuka, K. Minami, S. Tejima, and H. Nakamura, Phys. Rev. B 71, 134515 (2005); S. Savel'ev, V. Yampolyskii, A. Rakhmanov, and F. Nori, Phys. Rev. B 72, 144515 (2005).

${ }^{9}$ L. N. Bulaevskii and A. E. Koshelev, Phys. Rev. Lett. 99,
057002 (2007)

${ }^{10}$ S. Savel'ev, A. L. Rakhmanov, and F. Nori, Phys. Rev. Lett. 94, 157004 (2005); S. Savel'ev, V. Yampol'skii, and F. Nori, ibid. 95, 187002 (2005); S. Savel'ev, A. L. Rakhmanov, and F. Nori, ibid. 98, 077002 (2007); S. Savel'ev, A. L. Rakhmanov, V. A. Yampol'skii, and F. Nori, Nat. Phys. 2, 521 (2006).

${ }^{11}$ M. H. Bae, H. J. Lee, and J. H. Choi, Phys. Rev. Lett. 98, 027002 (2007).

${ }^{12}$ L. Ozyuzer, A. E. Koshelev, C. Kurter, N. Gopalsami, Q. Li, M. Tachiki, K. Kadowaki, T. Yamamoto, H. Minami, H. Yamaguchi, T. Tachiki, K. E. Gray, W.-K. Kwok, and U. Welp, Science 318, 1291 (2007).

${ }^{13}$ X. Y. Jin, J. Lisenfeld, Y. Koval, A. Lukashenko, A. V. Ustinov, and P. Müller, Phys. Rev. Lett. 96, 177003 (2006).

${ }^{14}$ H. J. H. Smilde, A. A. Golubov, Ariando, G. Rijnders, J. M. Dekkers, S. Harkema, D. H. A. Blank, H. Rogalla, and H. Hilgenkamp, Phys. Rev. Lett. 95, 257001 (2005).

${ }^{15}$ M. B. Gaifullin, K. Hirata, S. Ooi, S. Savel'ev, Yu. I. Latyshev, 
and T. Mochiku, Physica C (to be published).

${ }^{16}$ J. M. Dekkers, G. Rijnders, S. Harkema, H. J. H. Smilde, H. Hilgenkamp, H. Rogalla, and D. H. A. Blank, Appl. Phys. Lett. 83, 5199 (2003).

${ }^{17}$ B. Chesca, D. Doenitz, T. Dahm, R. P. Huebener, D. Koelle, R.
Kleiner, Ariando, H. J. H. Smilde, and H. Hilgenkamp, Phys. Rev. B 73, 014529 (2006).

${ }^{18}$ B. Chesca, H. J. H. Smilde, and H. Hilgenkamp, Phys. Rev. B 77, 184510 (2008). 